\title{
VAMPIRE: Improved Method for Automated Center Lumen Line Definition in Atherosclerotic Carotid Arteries in CTA Data
}

\author{
H.A.F. Gratama van Andel, E. Meijering, A. van der Lugt, \\ H.A. Vrooman, and R. Stokking* \\ Biomedical Imaging Group Rotterdam \\ Erasmus MC - University Medical Center Rotterdam \\ Departments of Medical Informatics and Radiology \\ P.O. Box 1738, 3000 DR Rotterdam, The Netherlands \\ * Corresponding Author, Tel.: +31-10-4088187 \\ r.stokking@erasmusmc.nl
}

\begin{abstract}
We evaluate a new method, called VAMPIRE, for automated definition of a center lumen line in vessels in cardiovascular image data. VAMPIRE is based on detection of vessel-like structures by analyzing firstorder and second-order image derivatives combined with a minimal cost path algorithm. The image derivatives are obtained using Canny edge detection and an improved ridge filter for detecting elongated structures. We compared VAMPIRE with an established technique in a multi-observer evaluation study involving 40 tracings in slabbed MIP images of multislice CTA datasets of atherosclerotic carotid arteries. The results show that VAMPIRE, in comparison with the established technique, presents considerably more successful tracings and improved handling of stenosis, calcifications, multiple vessels and nearby bone structures. We conclude that VAMPIRE is highly suitable for automated path definition in vessels in (clinical) cardiovascular image data and we expect to improve VAMPIRE to yield paths that are adequate enough to directly serve as center lumen lines in accordance with standards provided by clinicians.
\end{abstract}

\section{Introduction}

Cardiovascular disease causes millions of deaths every year. Visualization and quantification of the vasculature in patients suspected of atherosclerosis is of great importance and determining the degree of stenosis in arteries is crucial when considering therapeutical options. Increasingly, magnetic resonance angiography (MRA) and computed tomography angiography (CTA), both three-dimensional (3D) imaging techniques, are being used for diagnostic purposes on vessels [1]. In general, measurements on the MRA and CTA data are performed manually, which is time consuming and highly user dependent [2]. This has motivated the development of automated methods for fast, robust and reproducible segmentation, quantification and visualization of vessels in CTA and MRA data of patients suspected of atherosclerosis. Automated quantification of a stenosis generally involves definition of a 
center lumen line (CLL), either directly, by path tracing or, indirectly, by first segmenting the lumen (or vessel). Here we focus on direct path tracing.

Several methods have been proposed and evaluated for automated tracing of blood vessels. Direct vessel tracking [3] applies a local search algorithm based on detection of the edges of the lumen. This method has difficulties in dealing with vessels of high curvature and vessels where the lumen area does not change gradually, such as in the transition to a stenosis or an aneurysm. Another class is based on filtering of vessellike structures [4-8] by convolving the image with Gaussian-derivative filters on different scales and subsequent analysis of the Hessian (second-order derivative matrix) at every pixel. The resulting information can be applied for definition of a CLL and/or segmentation of the vasculature. Definition of a CLL generally involves a minimal cost path algorithm [7] or a B-spline curve fitting procedure [6]. The high computational cost of the Hessian analysis on multiple scales in $3 \mathrm{D}$ data is a problem, which can be surpassed by performing the Hessian analysis locally instead of globally [8]. Unfortunately, this requires presegmentation of the vasculature based on thresholding, which can be a cause of errors. Alternatively, segmentation of blood vessels can be performed by statistical inference using a priori information of the vasculature as a tree-like structure and a Gaussian intensity profile of the vessels [9].

Typical problems reported in automated path tracing are areas with stenoses, aneurysms, calcifications, other vessels and bone. Given these problems, we have developed a new method for automated definition of a CLL in cardiovascular image data which is based on first-order (edges) as well as second-order (ridges) image features combined with a minimal cost path algorithm. The edges are obtained using Canny edge detection and the ridges by means of an improved steerable filter for detecting elongated image structures $[10,11]$. In this paper we present the results of an evaluation of our method for (clinical) cardiovascular image data.

\section{Materials and Methods}

Our method for automated definition of a CLL in vessels in cardiovascular image data is called 'VAMPIRE' (Vascular Analysis using Multiscale Paths Inferred from Ridges and Edges). It is based on an improved ridge filter enhancing elongated structures [10, 11]. This filter was initially applied for neurite tracing and uses a modified Hessian which, in theory, is more sensitive to elongated structures compared to techniques based on normal Hessian analysis such as described by Frangi $[6,12]$ (a visual comparison of the two ridge-filters is shown in Figure 1).

VAMPIRE uses a multiscale implementation of the ridge filter in combination with a Canny edge filter [13] to make the technique suitable for cardiovascular data. A cost image is calculated from the resulting edge and ridge information (as illustrated in Figure 2). With this cost image and the vector information obtained from the Hessian analysis (see Figure 3), a minimal cost path is calculated with an implementation of Dijkstra's shortest path algorithm [14] (see also [7]). 


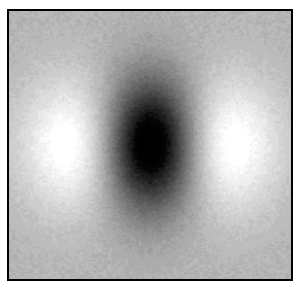

(a)

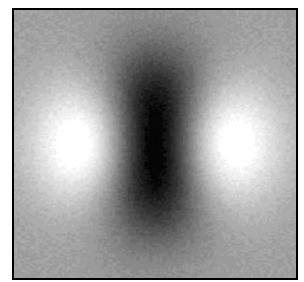

(b)

Fig. 1. Impression of the shapes of the used ridge filters: (a) filter based on normal Hessian analysis [12], (b) filter used in VAMPIRE. Clearly, the latter is more elongated and should, in theory, be more sensitive to vessel-like structures

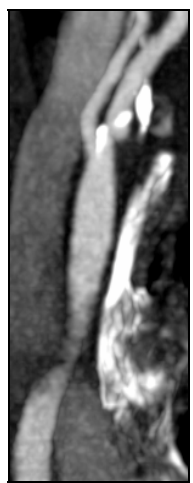

(a)

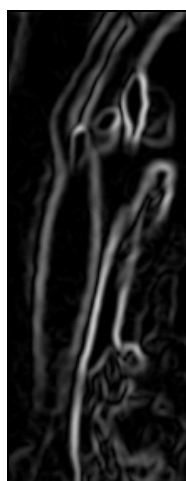

(b)

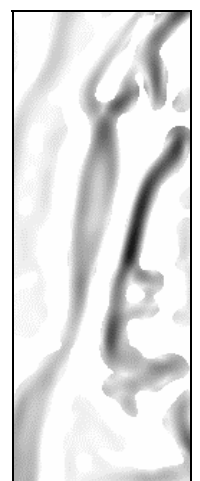

(c)

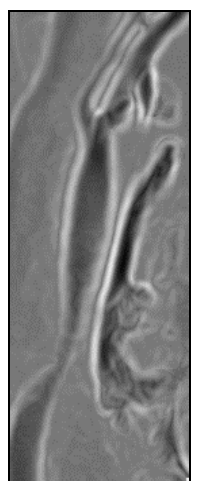

(d)

Fig. 2. Calculation of the cost image (d) from the original image (a) is based on the results of edge filtering (b) as well as ridge filtering (c)

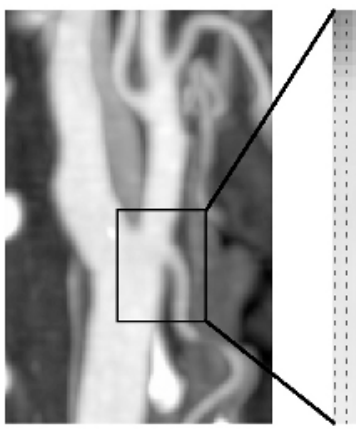

(a)

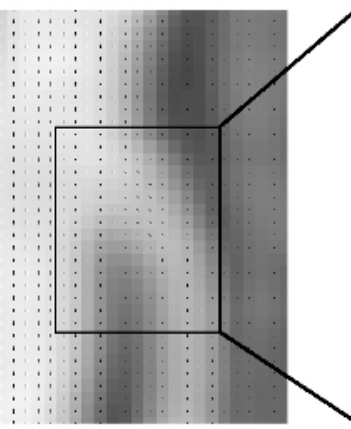

(b)

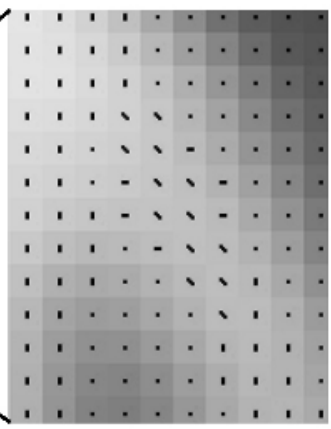

(c)

Fig. 3. The vector information computed with the Hessian analysis from (a) is shown in (b) and (c) as black lines for each pixel 
We compare VAMPIRE to a method based on the filter using normal Hessian analysis as described by Frangi et al. [12] for the enhancement of vessels, combined with a minimal cost path algorithm (same algorithm as VAMPIRE) as described by Wink et al. [7]. In the sequel, we refer to this latter method as the Frangi-Wink method. Both methods were implemented in $\mathrm{Java}^{\mathrm{TM}}[15]$ in the form of a plugin for use with ImageJ [16], a public domain image processing program.

The data used for the evaluation are 21 "slabbed" (volume of interest) maximum intensity projections (MIPs) of multislice CTA datasets from 14 patients with internal carotid artery (ICA) stenosis. These MIPs showed the bifurcation of the common carotid artery (CCA), the ICA with the stenosis and (part of) the external carotid artery (ECA) yielding a total of 40 CLLs (24 ICA paths and 16 ECA paths).

As reference, four qualified observers performed manual path tracings by placing points in the image, which were automatically connected by straight lines. The path tracings had to start as early as possible in the CCA and follow the ICA or ECA as long as possible. The paths were stored for further analysis and the mean tracing of the four observers for each vessel was calculated. In addition, the required time and number of points were registered so as to further evaluate the benefits of automating the definition of a CLL. The datasets were blinded and offered to the observers in different order to minimize distortion by learning effects.

As an intuitive measure of deviation of the automated tracings (VAMPIRE and the Frangi-Wink method) from the reference tracings (mean of the four observers) we used the area spanned between the automated tracing and corresponding reference tracing, divided by the length of the latter tracing (area/length).

\section{Results}

In Table 1 the results for the 40 path tracings are presented. The mean deviation of the paths manually defined by the four observers was $1.00 \mathrm{~mm}$. The deviation for each observer was assessed using the tracings of the other observers as reference. The mean deviation of VAMPIRE and the Frangi-Wink method was, respectively, $2.66 \mathrm{~mm}$ and $16.33 \mathrm{~mm}$. This difference is primarily caused by the fact that the path tracings obtained using the Frangi-Wink method left the target vessel in 29 out of 40 cases, compared to only 7 out of 40 cases with VAMPIRE.

Table 1. Deviations (in $\mathrm{mm}$ ) of all 40 path tracings based on 2 initialization points

\begin{tabular}{|l|c|c|c|c|c|c|c|}
\hline & $\begin{array}{c}\text { Obs. } \\
\mathbf{1}\end{array}$ & $\begin{array}{c}\text { Obs. } \\
\mathbf{2}\end{array}$ & $\begin{array}{c}\text { Obs. } \\
\mathbf{3}\end{array}$ & $\begin{array}{c}\text { Obs. } \\
\mathbf{4}\end{array}$ & $\begin{array}{c}\text { Manual } \\
\text { mean }\end{array}$ & VAMPIRE & $\begin{array}{c}\text { Frangi- } \\
\text { Wink }\end{array}$ \\
\hline Mean deviation & 1.08 & 1.02 & 0.90 & 0.99 & 1.00 & 2.66 & 16.33 \\
\hline Standard deviation & 0.33 & 0.29 & 0.29 & 0.33 & 0.31 & 2.32 & 19.91 \\
\hline
\end{tabular}

In Table 2, the results of VAMPIRE and the Frangi-Wink method are presented in three categories: (i) 'direct' tracings, initialized by one start and one end point that do not leave the vessel, (ii) 'direct \& extra click' tracings, requiring one additional point to stay in the vessel, and (iii) 'not in vessel' tracings, leaving the vessel even after an 
additional point is placed. In our opinion, the 'direct \& extra click' category can be considered to contain successful path definitions of the target vessel.

Table 2. Deviations (in $\mathrm{mm}$ ) in three categories

\begin{tabular}{|l|c|c|}
\hline Category & VAMPIRE & Frangi-Wink \\
\hline direct & $1.71 \mathrm{sd} .0 .51(\mathrm{n}=33)$ & $1.58 \mathrm{sd} .0 .64(\mathrm{n}=11)$ \\
\hline direct \& extra click & $1.70 \mathrm{sd} .0 .52(\mathrm{n}=40)$ & $1.61 \mathrm{sd} .0 .75(\mathrm{n}=20)$ \\
\hline not in vessel & - & $7.06 \mathrm{sd} .4 .28(\mathrm{n}=20)$ \\
\hline
\end{tabular}

VAMPIRE successfully defined a path in the target vessel in all 40 cases including only 7 cases requiring an additional point. In 20 out of 40 cases the Frangi-Wink method successfully defined a path in the desired vessel including 9 cases requiring an additional point. Figure 4 presents cases where the Frangi-Wink method resulted in paths leaving the target vessel because of bone, calcifications, or another vessel. The paths defined using VAMPIRE did not leave the target vessel. Both methods were initialized with the same start and end point. Figure 5 presents cases indicating three problems of the current implementation of VAMPIRE in defining a CLL.

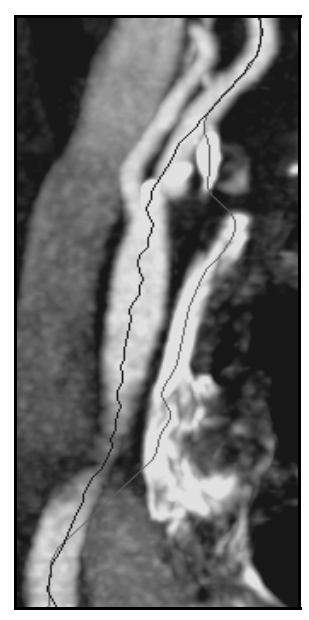

(a)

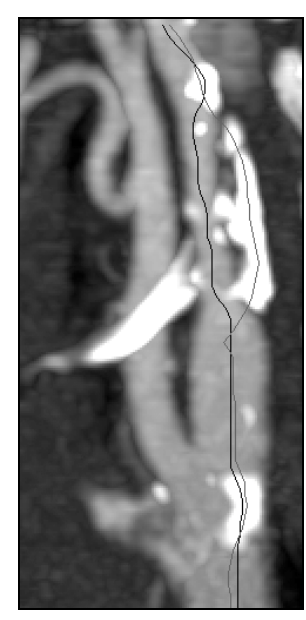

(b)

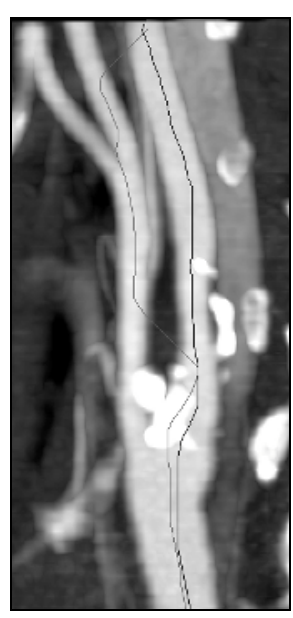

(c)

Fig. 4. Three examples of tracings in which the Frangi-Wink method (gray tracings) resulted in gross errors in path definition because it was attracted to (a) bone, (b) calcifications, or (c) another vessel. Paths defined using VAMPIRE (black tracings) did not leave the target vessels

The average time required for manual vessel tracing in these MIP images was 42 seconds and the average number of points used was 16. The time needed for calculation of the cost-image (essentially preprocessing) was 12 seconds on a Pentium IV $2 \mathrm{GHz}$, after which a minimal cost path was calculated almost instantly. In the 40 cases it took an average of 2-4 seconds for path-definition using VAMPIRE. 


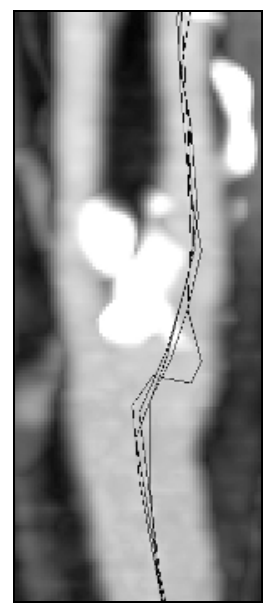

(a)

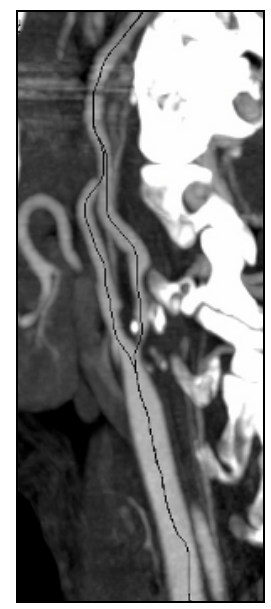

(b)

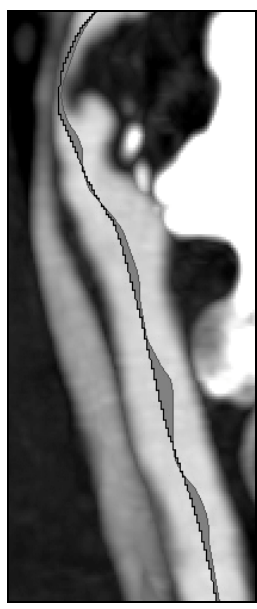

(c)

Fig. 5. Three examples of problems with definition of a CLL: (a) tracings of a CLL by multiple observers, (b) a VAMPIRE tracing through the wrong vessel caused by the proximity of vessels in the image, and (c) occasional swaying behavior in the tracings defined by VAMPIRE

\section{Discussion}

As a proof of concept we decided to first evaluate a 2D implementation of VAMPIRE on clinical data. This allowed us to save considerably on time and effort, not only in implementing and testing the method, but also in the manual definition of paths by the clinicians. Our results show that VAMPIRE, in comparison with the Frangi-Wink method, presents considerably more successful tracings and improved handling of stenosis, calcifications, multiple vessels and nearby bone structures. However, the design of the present study prevents a conclusion whether these improvements are primarily caused by the use of an improved ridge filter or by the addition of edge information. The Frangi-Wink method yielded a slightly lower mean deviation and a higher standard deviation in the successful tracings $(1.61 \mathrm{~mm}$ sd. 0.75$)$ compared to VAMPIRE (1.70mm sd. 0.52). The difference in mean deviation is probably due to the swaying behavior (see Figure 5(c)) of the tracings by VAMPIRE, which appears to result from multiple factors (edges in the lumen caused by inhomogeneous contrast filling, edges of the vessel itself, calcifications and nearby bone structures). The current version of VAMPIRE makes use of thresholding as a way to suppress edge responses in the lumen, but we expect to dispense with this step in the future. The differences in standard deviation indicate that VAMPIRE results in more consistent tracings than the Frangi-Wink method.

Additional experiments were carried out to test the applicability of our method on images in other clinical cardiovascular applications. Results of VAMPIRE for these cases are depicted in Figure 6. VAMPIRE was successful in finding a path through the vessels upon initialization of a startpoint and endpoint. 


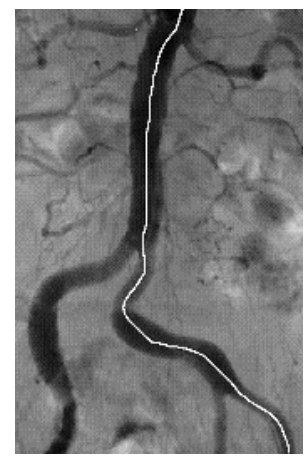

(a)

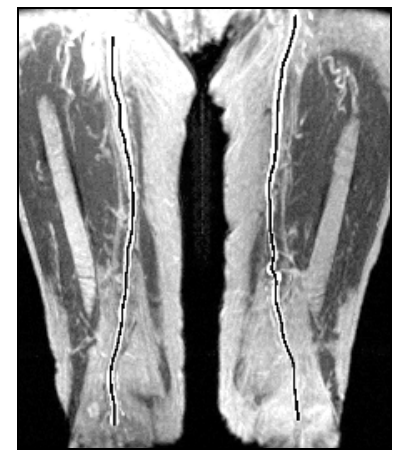

(b)

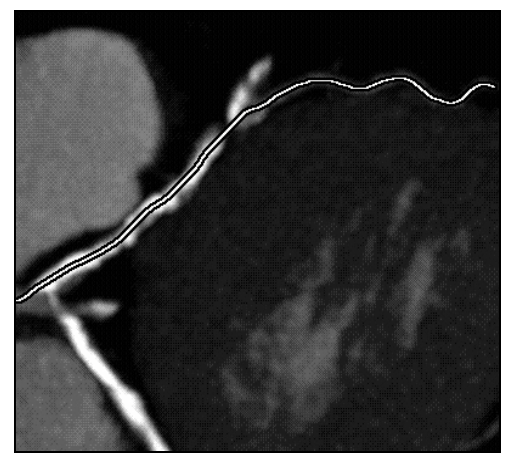

(c)

Fig. 6. Examples of tracings with VAMPIRE in (a) a DSA of aorta-iliac arteries, (b) a MIP of an MRA of the peripheral arteries, and (c) a MIP of a CTA of the coronary arteries

While it may be possible to uniquely (mathematically) define a CLL in simulated and phantom data of vessels, there is no unique way of defining the CLL of a vessel in clinical cases. The latter was exemplified by the variation in the paths manually defined by the observers (all trained in the same department), especially in areas involving e.g. a bifurcation, stenosis, or calcification (see the manually defined paths in Figure 5(a)). In addition, the definition of an 'optimal' CLL (or path) may be dependent on the clinical question at hand: for example, the optimal path defined for stent placement may be different from the path defined for quantification of stenoses. In order to further improve VAMPIRE, one of the key issues will be to develop standards for CLL definition in clinical cases.

We emphasize our finding that the dependency on the observer in tracing a path with VAMPIRE is virtually absent. In the MIP images of the carotids only two or three points were required and it is easy to use those points as a first guess, whereupon VAMPIRE is able to define the optimal points based on the available edge and ridge information (snapping). This means that no observer-critical decision is required to define a path in these images. In the future, the remaining observer input can be replaced by using an atlas or (CLL) model (e.g. based on statistical analysis) in combination with nonlinear registration so as to provide the relevant initialization data. This would result in completely user-independent vessel tracing and the observer will only be required to verify the results of the path tracing.

We conclude that the VAMPIRE approach has a high potential for automated path definition in vessels in (clinical) cardiovascular image data and we expect to further develop the technique to yield paths (also in 3D) that may directly serve as CLLs in accordance with standards provided by clinicians. 


\section{References}

[1] P. J. Nederkoorn, W. P. Th. M. Mali, B. C. Eikelboom, O. E. Elgersma, E. Buskens, M. G. Hunink, L. J. Kappelle, P. C. Buijs, A. F. Wust, A. van der Lugt, Y. van der Graaf. Preoperative Diagnosis of Carotid Artery Stenosis: Accuracy of Noninvasive Testing. Stroke 33(8):2003-8, 2002.

[2] G. D. Rubin. Data Explosion: The Challenge of Multidetector-Row CT. European Journal of Radiology 36(2):74-80, 2000.

[3] O. Wink, W. J. Niessen, M. A. Viergever. Fast Delineation and Visualization of Vessels in 3-D Angiographic Images. IEEE Transactions on Medical Imaging 19(4):337-46, 2000 .

[4] Y. Sato, S. Nakajima, N. Shiraga, H. Atsumi, S. Yoshida, T. Koller, G. Gerig, R. Kikinis. Three-Dimensional Multi-Scale Line Filter for Segmentation and Visualization of Curvilinear Structures in Medical Images. Medical Image Analysis 2(2):143-68, 1998.

[5] C. M. van Bemmel, L. Spreeuwers, M. A. Viergever, W. J. Niessen. Central Axis Based Segmentation of the Carotids. In: Proceedings of the International Society of Magnetic Resonance in Medicine, ISMRM-2002, p. 1797.

[6] A. F. Frangi, W. J. Niessen, R. M. Hoogeveen, T. van Walsum, M. A. Viergever. ModelBased Quantitation of 3-D Magnetic Resonance Angiographic Images. IEEE Transactions on Medical Imaging 18(10):946-56, 1999.

[7] O. Wink, A. F. Frangi, B. Verdonck, M. A. Viergever, W. J. Niessen. 3D MRA Coronary Axis Determination using a Minimum Cost Path Approach. Magnetic Resonance Imaging 47(6):1169-75, 2002.

[8] S. R. Aylward, E. Bullitt. Initialization, Noise, Singularities, and Scale in Height Ridge Traversal for Tubular Object Centerline Extraction. IEEE Transactions on Medical Imaging 21(2):61-75, 2002.

[9] A. Bhalerao, E. Thönnes, W. Kendall, R. Wilson. Inferring Vascular Structure from 2D and 3D Imagery. In: Proceedings of Medical Image Computing and Computer-Assisted Intervention, MICCAI-2001, pp 820-828.

[10] M. Jacob, M. Unser. Design of Steerable Filters for Feature Detection using Canny-Like Criteria. IEEE Transactions on Pattern Analysis and Machine Intelligence, 2004. In press.

[11] E. Meijering, M. Jacob, J.-C. F. Sarria, P. Steiner, H. Hirling, M. Unser. Design and Validation of a Tool for Neurite Tracing and Analysis in Fluorescence Microscopy Images. Cytometry, 2004. In press.

[12] A. F. Frangi, W. J. Niessen, K. L. Vincken, M. A. Viergever. Mutiscale Vessel Enhancement Filtering. In: Proceedings of Medical Image Computing and ComputerAssisted Intervention, MICCAI-1998, pp. 130-137.

[13] J. Canny. A Computational Approach to Edge Detection. IEEE Transactions on Pattern Analysis and Machine Intelligence 8(6):679-698, 1986.

[14] T. H. Cormen, C. E. Leiserson, R. L. Rivest, C. Stein. Introduction to Algorithms. $2^{\text {nd }}$ edition. Cambridge, MA, MIT Press, 2001.

[15] Sun Microsystems, Inc. Java ${ }^{\mathrm{TM}}$ Platform Standard edition, http://java.sun.com/

[16] W. Rasband. ImageJ: Image Processing and Analysis in Java. U.S. National Institutes of Health, http://rsb.info.nih.gov/ij/ 